\title{
The anti-biofilm effect of silver-nanoparticle-decorated quercetin nanoparticles on a multi-drug resistant Escherichia coli strain isolated from a dairy cow with mastitis
}

\author{
Lumin Yu ${ }^{1}$, Fei Shang ${ }^{1}$, Xiaolin Chen ${ }^{1}$, Jingtian $\mathbf{N i}{ }^{1}$, Li Yu ${ }^{2}$, Ming Zhang ${ }^{1}$, Dongdong Sun ${ }^{1}$, Ting Xue \\ Corresp. 1 \\ ${ }^{1}$ School of Life Sciences, Anhui Agricultural University, Hefei, Anhui, China \\ 2 Department of Microbiology and Parasitology, Anhui Key Laboratory of Zoonoses, Anhui Medical University, Hefei, Anhui, China \\ Corresponding Author: Ting Xue \\ Email address: xuet@ahau.edu.cn
}

Background Escherichia coli is an important opportunistic pathogen that could cause inflammation of the udder in dairy cows resulting in reduced milk production and changes in milk composition and quality, and even death of dairy cows. Therefore, mastitis is the main health issue which leads to major economic losses on dairy farms. Antibiotics are routinely used for the treatment of bovine mastitis. The ability to form biofilm increases the antibiotic resistance of $E$. coli. Nanoparticles (NPs), a nanosized, safe, and highly costeffective antibacterial agent, are potential biomedical tools. Given their antibacterial activities, silver nanoparticles (Ag NPs) have a broad range of applications. Methods In this study, we performed antibacterial activity assays, biofilm formation assays, scanning electron microscopy (SEM) experiments, and real-time reverse transcription PCR (RT-PCR) experiments to investigate the antibacterial and anti-biofilm effect of quercetin, Ag NPs, and Silver-nanoparticle-decorated quercetin nanoparticles (QA NPS) in E. coli strain ECDCM1. Results In this study, QA NPs, a composite material combining Ag NPs and the plant-derived drug component quercetin, exhibited stronger antibacterial and anti-biofilm properties in a multi-drug resistant $E$. coli strain isolated from a dairy cow with mastitis, compared to Ag NPs and Qe. Discussion This study provides evidence that QA NPS possess high antibacterial and anti-biofilm activities. They proved to be more effective than Ag NPs and Qe against the biofilm formation of a multi-drug resistant $E$. coli isolated from cows with mastitis. This suggests that QA NPs might be used as a potential antimicrobial agent in the treatment of bovine mastitis caused by $E$. coli. 
1 RUNNING HEAD: The anti-biofilm effect of QA NPs on $E$. coli

2

3 The anti-biofilm effect of silver-nanoparticle-decorated quercetin nanoparticles on a multi-

4 drug resistant Escherichia coli strain isolated from a dairy cow with mastitis

5

6 Lumin Yu*, Fei Shang*, Xiaolin Chen*, Jingtian Ni*, Li Yut, Ming Zhang*, Dongdong

7 Sun*1, and Ting Xue ${ }^{* 1}$

8

9 *School of Life Sciences, Anhui Agricultural University, Hefei, Anhui 230036, China

$10 \dagger^{\dagger}$ Department of Microbiology and Parasitology, Anhui Key Laboratory of Zoonoses, Anhui

11 Medical University, Hefei 230032, China

13 Lumin Yu and Fei Shang have contributed equally to this study.

$15{ }^{1}$ Corresponding author:

E-mail: sunddwj@126.com, Tel: 86-551-65787380 


\section{ABSTRACT}

24

Background Escherichia coli is an important opportunistic pathogen that could cause inflammation of the udder in dairy cows resulting in reduced milk production and changes in milk composition and quality, and even death of dairy cows. Therefore, mastitis is the main health issue which leads to major economic losses on dairy farms. Antibiotics are routinely used for the treatment of bovine mastitis. The ability to form biofilm increases the antibiotic resistance of E. coli. Nanoparticles (NPs), a nanosized, safe, and highly cost-effective antibacterial agent, are potential biomedical tools. Given their antibacterial activities, silver nanoparticles (Ag NPs) have a broad range of applications.

Methods In this study, we performed antibacterial activity assays, biofilm formation assays, scanning electron microscopy (SEM) experiments, and real-time reverse transcription PCR (RTPCR) experiments to investigate the antibacterial and anti-biofilm effect of quercetin, Ag NPs, and Silver-nanoparticle-decorated quercetin nanoparticles (QA NPs) in E. coli strain ECDCM1.

Results In this study, QA NPs, a composite material combining Ag NPs and the plant-derived drug component quercetin, exhibited stronger antibacterial and anti-biofilm properties in a multidrug resistant $E$. coli strain isolated from a dairy cow with mastitis, compared to Ag NPs and Qe.

Discussion This study provides evidence that QA NPs possess high antibacterial and anti-biofilm activities. They proved to be more effective than Ag NPs and Qe against the biofilm formation of a multi-drug resistant $E$. coli isolated from cows with mastitis. This suggests that QA NPs might be used as a potential antimicrobial agent in the treatment of bovine mastitis caused by E. coli. 
65

66

67

Mastitis, an infection or inflammation of the parenchyma of the mammary gland, is considered to be one of the most frequent and troublesome diseases in dairy cows and results in significant economic losses to the dairy industry worldwide (Halasa et al. 2007; Miller et al. 1993). Escherichia coli may cause mastitis which can result in death due to severe endotoxaemia (Kempf et al. 2016).

Antimicrobials are used frequently for treatment and prevention of clinical E. coli mastitis in cows (Srinivasan et al. 2007). However, due to the extensive use of antibacterial agents in bovine mastitis, the emergence of antibiotic-resistant bacteria and the frequent reoccurrence of chronic bovine mastitis has become a critical issue (Chambers 2001; Gomes \& Henriques 2016). Another major reason for antibiotic resistance and recurrence of inflammation is bacterial biofilm formation inside the udder tissue (Atulya et al. 2014; Davies 2003; Olson et al. 2002). Biofilms are defined as communities of microorganisms growing on biotic and abiotic surfaces that tightly adhered to each other in a self-produced extracellular matrix of exopolysaccharide (EPS), proteins, and DNA (Costerton et al. 1987; Prigent-Combaret et al. 2001). In E. coli, various cell surface appendages such as flagella, type I fimbriae, and curli as well as the production of the EPS (such as cellulose, colanic acid, etc.) participate in early adhesion steps (Cookson et al. 2002; Pratt \& Kolter 1998). During the maturation of the biofilm, multiplication of bacterial cells cause an expansion of the microcolonies (Prigent-Cmbaret et al. 2000; Reisner et al. 2003; Srinivasan et al. 2007). Therefore, biofilms can become hundreds of micrometers in depth and display 
85

complex structural and functional architecture (Costerton et al. 1987; Pratt \& Kolter 1998;

Prigent-Cmbaret et al. 2000; Prigent-Combaret et al. 2001). The biofilm can protect E. coli against antibiotic therapy and host defense in bovine mastitis (Dantas et al. 2008; Melchior et al. 2006; Xue et al. 2014). Therefore, the capacity of $E$. coli to form biofilms can result in persistent inflammatory infection in the bovine mammary gland, which is difficult to prevent, control, or eradicate (Labbate et al. 2004; Ren et al. 2005).

In recent years, nanoparticles, safe, cost effective bactericidal materials, which are gaining popularity, and have been used successfully as carriers of therapeutic agents, in diagnostics of chronic diseases (Gomes \& Henriques 2016; Hong et al. 2008; Martinez-Gutierrez et al. 2013; Zhang et al. 2008). In addition, although silver is toxic to microorganisms, it is less dangerous to mammalian cells than other metals (Morones et al. 2005; Sondi \& Salopeksondi 2004; Sun et al. 2017; Zhao \& Jr 1998). Silver nanoparticles (Ag NPs or nanosilver), a kind of nanosized silver particle, are widely used NPs and show strong biocidal effects on a broad spectrum of bacterial pathogens, including E. coli (Morones et al. 2005; Sondi \& Salopeksondi 2004). However, Choi et al. (Choi et al. 2010) demonstrated that biofilm formation in E. coli significantly increased resistance to Ag NPs. Hence, the synergistic effect of Ag NPs and other antimicrobial agents, especially plant-derived drugs, has gotten a lot of attention (Gomes \& Henriques 2016). Previous reports show that plant-derived drugs have the advantage of not inducing resistance after prolonged exposure and are easily obtained (Domadia et al. 2007; Ohno et al. 2003; Sun et al. 2017). 3,3',4',5,7-Pentahydroxyflavone (quercetin, Qe), a kind of flavonoid compound, is 
106

107

108

109

110

111

112

113

114

115

116

117

118

119

120

121

122

123

124

125

126

commonly present in various foods including onions, fruits, and vegetables (Smith et al. 2016;

Sun et al. 2017). Silver-nanoparticle-decorated quercetin nanoparticles (QA NPs) showed highly effective antibacterial activity against drug-resistant E. coli (Sun et al. 2017). However, whether QA NPs affect biofilm formation of E. coli has not been reported. In this study, we examined the effects of QA NPs on growth and biofilm formation of E. coli isolated from a dairy cow with mastitis, and explored the regulatory role of QA NPs in biofilm-associated gene expression.

\section{MATERIALS AND METHODS}

\section{Strains and Materials}

The multi-drug resistant E. coli strain ECDCM1 used in this study was isolated from a dairy cow with mastitis. Strain ECDCM1 was stored at $-80{ }^{\circ} \mathrm{C}$. Before each experiment, it was first cultured on Luria-Bertani (LB) agar plates which contained 10 g/L Bacto Tryptone (Oxoid, Basingstoke, UK), 5 g/L yeast extract (Oxoid, Basingstoke, UK), 10 g/L NaCl (Sangon, Shanghai, China), and $20 \mathrm{~g} / \mathrm{L}$ agar powder (Sangon, Shanghai, China) for $16 \mathrm{~h}$ at $37{ }^{\circ} \mathrm{C}$ in air supplied with $5 \% \mathrm{CO}_{2}$. QA NPs were synthesized according to a previous study (Sun et al. 2017).

\section{Antibacterial activity assays}

Antibacterial activity assays were performed according to the following methods (Chen et al. 2015). Colonies of strain ECDCM1 were placed into $3 \mathrm{~mL}$ of Mueller-Hinton (MH) broth (Oxoid, Basingstoke, UK) and cultivated at $37{ }^{\circ} \mathrm{C}$ with shaking at $180 \mathrm{rpm}$ for $16 \mathrm{~h}$. The overnight cultures were inoculated into fresh $\mathrm{MH}$ broth and diluted to a final concentration (600 $\mathrm{nm}$ ) of 0.03 before being dispensed into 96-well plates (Costar, Corning, Steuben, NY) 
127

128

129

130

131

132

133

134

135

136

137

138

139

140

141

142

143

144

145

146

147

containing Qe, Ag NPs, or QA NPs at the concentrations from $0 \mathrm{mg} / \mathrm{mL}$ to $10 \mathrm{mg} / \mathrm{mL}$, respectively. The bacteria with or without addition of Qe, Ag NPs, and QA NPs were incubated

at $37{ }^{\circ} \mathrm{C}$ for $20 \mathrm{~h}$, and then 10 -fold serial dilutions of cultures were performed by successive transfer $(0.1 \mathrm{~mL})$ through six microfuge tubes containing $0.9 \mathrm{~mL}$ of $\mathrm{MH}$. Next, $100 \mu \mathrm{L}$ dilutions were dropped on $\mathrm{MH}$ agar plates. After cultivating for $16 \mathrm{~h}$ at $37^{\circ} \mathrm{C}$, viable colonies were counted and compared between the control and test groups via their colony-forming units on $\mathrm{MH}$ agar plates. The survival rates of the control groups without exposure to Qe, Ag NPs, or QA NPs were designated as $100 \%$. Experiments were repeated three times with four parallels.

\section{Biofilm Formation Assay}

Biofilm formation in sterile 96 -well plates or $15 \mathrm{~mm} \times 150 \mathrm{~mm}$ glass tubes was produced as described previously and modified as follows (Chen et al. 2015; Rezaei et al. 2013). Briefly, E. coli strain ECDCM1 was grown for $16 \mathrm{~h}$ in LB medium and diluted to a ratio of 1:50 into fresh LB medium containing $0.5 \%$ milk solution. The different concentrations of Qe, Ag NPs, and QA NPs, respectively, were added to the LB medium with $0.5 \%$ milk solution and $E$. coli cells. The contents were then transferred into 96-well plates and $15 \mathrm{~mm} \times 150 \mathrm{~mm}$ glass tubes and incubated without shaking at $37{ }^{\circ} \mathrm{C}$ for $72 \mathrm{~h}$ and $48 \mathrm{~h}$, respectively. After incubation, the growth medium was decanted, and the wells were washed three times with sterile phosphate buffered saline (PBS pH 7.4) and air-dried. On observation the biofilms attached to the glass tubes were photographed. In addition, the biofilms attached to 96-well plates were determined quantitatively using crystal violet assay (Chen et al. 2015; O'Toole 2011). First, the biofilm-formation cells were fixed with $100 \%$ methanol for 5 min. After decanting the methanol, the wells were air-dried 
148 and stained for $15 \mathrm{~min}$ with $0.1 \%(\mathrm{w} / \mathrm{v})$ crystal violet (CV, Sangon, Shanghai, China). Next, 149 excess stain was gently rinsed with distilled water and air-dried overnight. Finally, the CV 150 remaining in the wells was dissolved in 33\% glacial acetic acid (Sangon, Shanghai, China) and 151 was subsequently read using a MicroELISA autoreader (Thermo Scientific, Pittsburgh, PA) at a 152 wavelength of $492 \mathrm{~nm}$ in single wavelength mode. Absorbance data from three replicate wells 153 were averaged to obtain each data point.

154 Scanning Electron Microscopy

155 Scanning electron microscopy (SEM, XL20, Philips, Holland) was used to investigate the 156 structural modifications of biofilms after treatment with Qe, Ag NPs, or QA NPs. For biofilm 157 formation, the overnight $E$. coli strain ECDCM1 was diluted to a ratio of 1:50 into fresh LB 158 medium complemented with $0.5 \%$ milk solution. The cultures were added to 12 -well plates 159 (Costar, Corning, Steuben, NY), when necessary, Qe, Ag NPs, or QA NPs were added for a final concentration of $5 \mu \mathrm{g} / \mathrm{mL}$. Sterile $18 \mathrm{~mm} \times 18 \mathrm{~mm}$ coverslips were placed in the wells and served as the attaching surface for the cells. The plates were incubated for $15 \mathrm{~h}$ at $37{ }^{\circ} \mathrm{C}$. After incubation, the coverslips were taken out and washed three times with PBS (pH 7.4). The preparation of the samples for SEM was performed as follows(Sun et al. 2017). The samples subsequently dehydrated using serial ethanol concentrations: 30, 50, 70, 80, 95 and 100\%. Each 167 overnight. 
169 The transcript levels of the biofilm-formation related genes, which are $b c s A$ (encoding the 170 catalytic subunit of bacterial cellulose synthase), $\operatorname{csg} A$ (encoding the major subunit of curli 171 fibers), fliC (encoding the basic subunit of $E$. coli flagellar filament structural protein), fimA 172 (encoding the major subunit of $E$. coli type 1 fimbriae), motA (encoding a protein that enables 173 flagellar motor rotation), and $w c a F$ (encoding a putative acetyltransferase involved in colanic 174 acid biosynthesis) were tested by performing real-time reverse transcription-PCR experiments. 175 First, overnight cultures of E. coli strain ECDCM1 were diluted to a ratio of 1:50 into fresh LB medium complemented with $0.5 \%$ milk solution and $5 \mu \mathrm{g} / \mathrm{mL}$ of Qe, Ag NPs, or QA NPs were added. The contents were transferred to 12 -well plates and grown to the exponential phase at $37{ }^{\circ} \mathrm{C}$ without shaking. E. coli cells were then collected by centrifugation and resuspended in TrisEDTA buffer ( $\mathrm{pH} 8.0)$ containing $10 \mathrm{~g} / \mathrm{L}$ lysozyme. After incubation for $5 \mathrm{~min}$ at $37{ }^{\circ} \mathrm{C}$, total RNA was extracted from cells using the Trizol reagent (Ambion, Austin, TX) and residual DNA was removed using DNaseI (TaKaRa, Dalian, China). Next, real-time RT-PCR was performed using the PrimeScript 1st Strand cDNA synthesis kit, SYBR Premix Ex Taq (TaKaRa, Dalian, China), and a StepOne real-time PCR system (Applied Biosystems, Carlsbad, CA). Last, differences of gene expression were calculated by $\triangle \triangle \mathrm{Ct}$ (where $\mathrm{Ct}=$ cycle threshold) method using the $16 \mathrm{~S}$ rDNA gene as the housekeeping gene, normalized by subtracting the $\mathrm{Ct}$ value of $16 S$ cDNA from target cDNA. All of the real-time RT-PCR assays were repeated at least three times with similar results. The primers used in this study are listed in Table 1, and the PCR amplification efficiency was controlled between 1.93 and 2.09.

\section{Statistical Analysis}


190

194

195

196

197

198

199

200

201

202

203

204

205

206

207

208

209

210

All data were analyzed using the statistical software SPSS (ver. 19.0, IBM Corp., Armonk, NY)

by a one-way ANOVA method; the test results were shown as mean $\pm \mathrm{SD}$. The paired t-test was used for statistical comparisons between groups. The level of statistical significance was set at a $\mathrm{P}$-value of $\leq 0.05$.

\section{Antibacterial Effect of QA NPs on E. coli Strain ECDCM1}

Since QA NPs were synthesized with the raw material Qe and Ag NPs, the survival rates of $E$. coli strain ECDCM1 exposed to Qe and Ag NPs were firstly detected. As shown in Fig. 1A and $\mathrm{B}$, Qe and Ag NPs did not affect the survival rates of $E$. coli strain ECDCM1 at concentrations of $0.5 \mu \mathrm{g} / \mathrm{mL}, 1 \mu \mathrm{g} / \mathrm{mL}$, and $5 \mu \mathrm{g} / \mathrm{mL}$; and when the concentrations of Qe and Ag NPs reached 10 $\mu \mathrm{g} / \mathrm{mL}$, the survival rates of $E$. coli strain ECDCM1 were approximately $74 \%$ and $52 \%$, respectively, compared with the control group without exposure to Qe or Ag NPs. However, except for the $0.5 \mu \mathrm{g} / \mathrm{mL}$ concentration of QA NPs, the survival rate of E. coli strain ECDCM1 decreased accordingly with the increase of the concentration of QA NPs, and when the concentration of QA NPs reached $10 \mu \mathrm{g} / \mathrm{mL}$, the survival rate of cells dropped to $0 \%$. These data indicated that QA NPs had a higher antibacterial activity against E. coli strain ECDCM1 than Qe and Ag NPs.

\section{Inhibitory Effect of QA NPs on Biofilm Formation of E. coli Strain ECDCM1}

The biofilm was formed both in glass tubes and in a 96-well plate to examine the influence of QA NPs on biofilm formation capacity of E. coli strain ECDCM1. As shown in Fig. 2A, B and C, 
211 Qe, Ag NPs, and QA NPs have no apparent effect on biofilm formation of E. coli strain

212 ECDCM1 at concentrations of $1 \mu \mathrm{g} / \mathrm{mL}$ and $5 \mu \mathrm{g} / \mathrm{mL}$, and at concentration of $10 \mu \mathrm{g} / \mathrm{mL}$, biofilm

213 formation shows only a slight decrease; however, when the concentration reached $20 \mu \mathrm{g} / \mathrm{mL}$,

214 biofilm formation is highly inhibited. Especially, at the concentration of $20 \mu \mathrm{g} / \mathrm{mL}$, QA NPs

215 almost inhibited biofilm formation of E. coli strain ECDCM1 on glass tubes to an invisible

216 extent. In addition, the quantity of biofilm formation on the 96-well plates was tested using a

217 MicroELISA autoreader (Thermo Scientific, Pittsburgh, PA). We found that biofilm quantity

218 significantly decreased with the increase of QA NPs compared with that of Ag NPs, and biofilm

219 quantity did not decrease until the concentration of Qe reached $20 \mu \mathrm{g} / \mathrm{mL}$ (Fig. 2D, E and F).

To further investigate the effect of QA NPs on the biofilm integrity of E. coli strain ECDCM1, we performed a SEM experiment. Results showed that biofilm of the control group were compact and integral (Fig. 3A). And when $5 \mu \mathrm{g} / \mathrm{mL}$ concentrations of different agents were added, biofilms were damaged to varying degrees. As shown in Fig. 3B, when the cultures are exposed to Qe, the biofilms are hardly damaged, and the biofilm forms were similar to that of the control group. Meanwhile, biofilms of the Ag NPs treatment group were only in slight surface crack (Fig. 3C). However, biofilms of the QA NPs treatment group were seriously damaged (Fig. 3D), indicating that QA NPs highly damaged the biofilm integrity of E. coli strain ECDCM1. These results illustrated that QA NPs exhibit stronger inhibition against biofilm formation of $E$. coli strain ECDCM1 than both Qe and Ag NPs. 
232 To further confirm the inhibitory effect of QA NPs on biofilm formation of E. coli strain

233 ECDCM1, the transcript levels of biofilm-associated genes $(b \operatorname{cs} A, \operatorname{csg} A, f l i C, f i m A, \operatorname{mot} A$, and

$234 w c a F$ ) were tested by performing real-time RT-PCR experiments. Results showed that the

235 transcript levels of $b \operatorname{cs} A, \operatorname{csg} A, f l i C$, fim $A$, mot $A$, and $w c a F$ were significantly decreased upon the

236 addition of Qe, Ag NPs, or QA NPs; moreover, with the addition of QA NPs, the transcript

237 levels of $b c s A, \operatorname{csg} A, f l i C$, fim $A, \operatorname{mot} A$, and $w c a F$ decreased to lower extents than with that of Qe

238 and Ag NPs (Fig. 4), indicating that the anti-biofilm effect of QA NPs was strongest among the

239 three antimicrobial agents. These results confirmed that QA NPs efficiently inhibited biofilm

240 formation of E. coli strain ECDCM1 by decreasing transcription of biofilm-associated genes

$241 b \operatorname{cs} A, \operatorname{csg} A$, fliC, fim $A, \operatorname{mot} A$, and $w c a F$.

\section{DISCUSSION}

244 Bacteria in biofilms usually induce chronic mastitis characterized by prolonged inflammation

245 and respond poorly to conventional treatments (Dean et al. 2011; Secor et al. 2011). The

246 antibiotic therapy is the important strategy for bovine mastitis treatment (Saini et al. 2012; Saini

247 et al. 2013; Yuan et al. 2017). However, due to the overuse and misuse of antibiotics, the

248 emergence of bacterial resistance among bacteria has become a serious problem in cows with

249 mastitis (Gomes \& Henriques 2016). Moreover, the biofilm formation is associated with the

250 increase of bacterial resistance to antibiotics (Dantas et al. 2008; Davies 2003). Hence, there is

251 an urgent need for inhibition of the bacterial biofilm formation for controlling bovine mastitis.

252 Plant-derived drugs are new biologically active agents with antimicrobial activity (Gomes \& 
253 Henriques 2016). For example, the plant extract ursolic acid inhibited biofilm formation of $E$. 254 coli (Ren et al. 2005), and the ethanol extract of Sanguisorba officinalis strongly inhibited the 255 biofilm formation of methicillin-resistant Staphylococcus aureus (MRSA) (Chen et al. 2015).

256 In this study, the strain we used is a clinical multidrug resistant $E$. coli strain which is resistant 257 to a variety of antibiotics, and also has a strong capacity to form a biofilm. This kind of strains 258 always causes infections which are difficult to cure. Our data showed that $1 \mu \mathrm{g} / \mathrm{mL}$ QA NPs 259 significantly inhibited the growth of strain ECDCM1. Furthermore, at concentration of $5 \mu \mathrm{g} / \mathrm{mL}$, 260 QA NPs can disintegrate the structure of biofilm and even kill bacteria in the biofilm. These data indicated that QA NPs was comparable to a kind of highly effective antibiotic. In addition, Ag

262 NPs, the most widely used NPs, and Qe, a plant-derived ingredient, are both readily available 263 and not expensive. Thus, the complex QA NPs might have high application value and deveolpment potential in therapy for bovine mastitis.

\section{CONCLUSIONS}

267

This study found that QA NPs, a composite material combining Ag NPs and the plant-derived drug component quercetin (Kareem \& Al-Hamadani 2016), can strongly inhibit the biofilm formation of an E. coli strain isolated from a dairy cow with mastitis by decreasing transcription of biofilm-associated genes $b \operatorname{cs} A, \operatorname{csg} A, \operatorname{fli}$, fim $A, \operatorname{mot} A$, and $w c a F$.

\section{ACKNOWLEDGMENTS}

272 The authors would like to thank Xiuhong Zhou for the technical assistance on scanning electron 273 microscope. 


\section{REFERENCES}

276

277

278

279

280

281

282

283

284

285

286

287

288

289

290

291

292

293

294

295

296

297

298

299

300

301

302

303

304

305

306

307

308

309

310

311

312

Atulya M, Mathew AJ, Rao JV, and Rao CM. 2014. Influence of milk components in establishing biofilm mediated bacterial mastitis infections in cattle: A fractional factorial approach. Research in Veterinary Science 96:25-27. DOI:10.1016/j.rvsc.2013.12.001

Chambers HF. 2001. The changing epidemiology of Staphylococcus aureus? Emerging Infectious Diseases 7:178182. DOI: $10.3201 /$ eid0702.010204

Chen X, Shang F, Meng Y, Li L, Cui Y, Zhang M, Qi K, and Xue T. 2015. Ethanol extract of Sanguisorba officinalis L. inhibits biofilm formation of methicillin-resistant Staphylococcus aureus in an ica-dependent manner. Journal of Dairy Science 98:8486-8491. DOI: 10.3168/jds.2015-9899

Choi OY, Yu CP, Fernández GE, and Hu ZQ. 2010. Interactions of nanosilver with Escherichia coli cells in planktonic and biofilm cultures. Water Research 44:6095-6103. DOI: 10.1016/j.watres.2010.06.069

Cookson AL, Cooley WA, and Woodward MJ. 2002. The role of type 1 and curli fimbriae of Shiga toxin-producing Escherichia coli in adherence to abiotic surfaces. International Journal of Medical Microbiology Ijmm 292:195-205. DOI: 10.1016/j.watres.2010.06.069

Costerton JW, Cheng KJ, Geesey GG, Ladd TI, Nickel JC, Dasgupta M, and Marrie TJ. 1987. Bacterial biofilms in nature and disease. Annual Review of Microbiology 41:435-464. DOI:10.1146/annurev.mi.41.100187.002251

Dantas G, Sommer MOA, Oluwasegun RD, and Church GM. 2008. Bacteria Subsisting on Antibiotics. Science 320:100-103. DOI: $10.1126 /$ science. 1155157

Davies D. 2003. Understanding biofilm resistance to antibacterial agents. Nature Reviews Drug Discovery 2:114122. DOI: $10.1038 / \operatorname{nrd} 1008$

Dean SN, Bishop BM, and Hoek MLV. 2011. Natural and synthetic cathelicidin peptides with anti-microbial and anti-biofilm activity against Staphylococcus aureus. Bmc Microbiology 11:114. DOI: 10.1186/1471-218011-114

Domadia P, Swarup S, Bhunia A, Sivaraman J, and Dasgupta D. 2007. Inhibition of bacterial cell division protein FtsZ by cinnamaldehyde. Biochemical Pharmacology 74:831-840. DOI: 10.1016/j.bcp.2007.06.029

Gomes F, and Henriques M. 2016. Control of Bovine Mastitis: Old and Recent Therapeutic Approaches. Current Microbiology 72:377-382. DOI: 10.1007/s00284-015-0958-8

Halasa T, Huijps K, O Ø, and Hogeveen H. 2007. Economic effects of bovine mastitis and mastitis management: a review. Veterinary Quarterly 29:18-31. DOI: 10.1080/01652176.2007.9695224

Hong B, Kai J, Ren Y, Han J, Zou Z, Chong HA, and Kang KA. 2008. Highly Sensitive Rapid, Reliable, and Automatic Cardiovascular Disease Diagnosis with Nanoparticle Fluorescence Enhancer and Mems. Advances in Experimental Medicine \& Biology 614:265-273. DOI: 10.1007/978-0-387-74911-2_30

Kareem AA, and Al-Hamadani AH. 2016. Synergistic Effects of Biosynthesized Nanoparticles Combined with Antibiotics against Pseudomonas aeruginosa and Proteus mirabilis. Journal of Applied Microbiology 2:128. DOI: $10.3389 /$ fmicb.2011.00128

Kempf F, Slugocki C, Blum SE, Leitner G, and Germon P. 2016. Genomic Comparative Study of Bovine Mastitis Escherichia coli. Plos One 11:e0147954. DOI: 10.1371/journal.pone.0147954 
313

314

315

316

317

318

319

320

321

322

323

324

325

326

327

328

329

330

331

332

333

334

335

336

337

338

339

340

341

342

343

344

345

346

347

348

349

350

351

352

353

Labbate M, Shu YQ, Kai SK, Rice SA, Givskov M, and Kjelleberg S. 2004. Quorum Sensing-Controlled Biofilm Development in Serratia liquefaciens MG1. Journal of Bacteriology 186:692-698. DOI: 10.1128/JB.186.3.692-698.2004

Martinez-Gutierrez F, Boegli L, Agostinho A, Bach H, Ruiz F, and James G. 2013. Anti-biofilm activity of silver nanoparticles against different microorganisms. Biofouling 29:651-660. DOI: $10.1080 / 08927014.2013 .794225$

Melchior MB, Vaarkamp H, and Fink-Gremmels J. 2006. Biofilms: a role in recurrent mastitis infections? Veterinary Journal 171:398-407. DOI: 10.1016/j.tvj1.2005.01.006

Miller GY, Bartlett PC, Lance SE, Anderson J, and Heider LE. 1993. Costs of clinical mastitis and mastitis prevention in dairy herds. Journal of the American Veterinary Medical Association 202:1230-1236.

Morones JR, Elechiguerra JL, Camacho A, Holt K, Kouri JB, Ramírez JT, and Yacaman MJ. 2005. The bactericidal effect of silver nanoparticles. Nanotechnology 16:2346-2353. DOI: 10.1088/0957-4484/16/10/059

O'Toole GA. 2011. Microtiter dish biofilm formation assay. Journal of Visualized Experiments Jove 47:e2437e2437. DOI: $10.3791 / 2437$

Ohno T, Kita M, Yamaoka Y, Imamura S, Yamamoto T, Mitsufuji S, Kodama T, Kashima K, and Imanishi J. 2003. Antimicrobial activity of essential oils against Helicobacter pylori. Helicobacter 8:207-215. DOI: 10.1046/j.1523-5378.2003.00146.x

Olson ME, Ceri H, Morck DW, Buret AG, and Read RR. 2002. Biofilm bacteria: formation and comparative susceptibility to antibiotics. Canadian Journal of Veterinary Research 66:86-92.

Pratt LA, and Kolter R. 1998. Genetic analysis of Escherichia coli biofilm formation: roles of flagella, motility, chemotaxis and type I pili. Molecular Microbiology 30:285-293. DOI:10.1046/j.1365-2958.1998.01061.x

Prigent-Cmbaret C, Prensier G, Le TT, Vidal O, Lejeune P, and Dorel C. 2000. Developmental pathway for biofilm formation in curli-producing Escherichia coli strains: role of flagella, curli and colanic acid. Environmental Microbiology 2:450-464. DOI: 10.1046/j.1462-2920.2000.00128.x

Prigent-Combaret C, Brombacher E, Vidal O, Ambert A, Lejeune P, Landini P, and Dorel C. 2001. Complex regulatory network controls initial adhesion and biofilm formation in Escherichia coli via regulation of the csgD gene. Journal of Bacteriology 183:7213-7223. DOI: 10.1128/JB.183.24.7213-7223.2001

Reisner A, Haagensen JA, Schembri MA, Zechner EL, and Molin S. 2003. Development and maturation of Escherichia coli K-12 biofilms. Molecular Microbiology 48:933-946. DOI: 10.1046/j.13652958.2003.03490.x

Ren D, Zuo R, González Barrios AF, Bedzyk LA, Eldridge GR, Pasmore ME, and Wood TK. 2005. Differential Gene Expression for Investigation of Escherichia coli Biofilm Inhibition by Plant Extract Ursolic Acid. Applied \& Environmental Microbiology 71:4022-4034. DOI: 10.1128/AEM.71.7.4022-4034.2005

Rezaei M, Moniri R, Mousavi SGA, and Shiade MJ. 2013. Prevalence of Biofilm Formation Among Methicillin Resistance Staphylococcus aureus Isolated From Nasal Carriers. Jundishapur Journal of Microbiology 6:e9601. DOI: 10.5812/jjm.9601

Saini V, Mcclure JT, Scholl DT, Devries TJ, and Barkema HW. 2012. Herd-level association between antimicrobial use and antimicrobial resistance in bovine mastitis Staphylococcus aureus isolates on Canadian dairy farms. Journal of Dairy Science 95:1921-1929. DOI: 10.3168/jds.2011-5065

Saini V, Mcclure JT, Scholl DT, Devries TJ, and Barkema HW. 2013. Herd-level relationship between antimicrobial use and presence or absence of antimicrobial resistance in gram-negative bovine mastitis pathogens on 
354

355

356

357

358

359

360

361

362

363

364

365

366

367

368

369

370

371

372

373

374

375

376

377

378

379

380

381

382

383

Canadian dairy farms. Journal of Dairy Science 96:4965-4976. DOI: 10.3168/jds.2012-5713

Secor PR, James GA, Fleckman P, Olerud JE, Mcinnerney K, and Stewart PS. 2011. Staphylococcus aureus Biofilm and Planktonic cultures differentially impact gene expression, mapk phosphorylation, and cytokine production in human keratinocytes. Bmc Microbiology 11:1-13. DOI: 10.1186/1471-2180-11-143

Smith AJ, Oertle J, Dan W, and Prato D. 2016. Quercetin: A Promising Flavonoid with a Dynamic Ability to Treat Various Diseases, Infections, and Cancers. Journal of Cancer Therapy 07:83-95. DOI: 10.4236/jct.2016.72010

Sondi I, and Salopeksondi B. 2004. Silver nanoparticles as antimicrobial agent: a case study on E. coli as a model for Gram-negative bacteria. Journal of Colloid \& Interface Science 275:177-182. DOI: 10.1016/j.jcis.2004.02.012

Srinivasan V, Gillespie BE, Lewis MJ, Nguyen LT, Headrick SI, Schukken YH, and Oliver SP. 2007. Phenotypic and genotypic antimicrobial resistance patterns of Escherichia coli isolated from dairy cows with mastitis. Veterinary Microbiology 124:319-328. DOI: 10.1016/j.vetmic.2007.04.040

Sun D, Zhang W, Mou Z, Chen Y, Guo F, Yang E, and Wang W. 2017. Transcriptome Analysis Reveals Silver Nanoparticle-Decorated Quercetin Antibacterial Molecular Mechanism. Acs Applied Materials \& Interfaces 9:10047-10060. DOI: 10.1021/acsami.7b02380

Xue T, Chen X, and Shang F. 2014. Short communication: Effects of lactose and milk on the expression of biofilmassociated genes in Staphylococcus aureus strains isolated from a dairy cow with mastitis. Journal of Dairy Science 97:6129-6134. DOI: 10.3168/jds.2014-8344

Yuan YG, Peng QL, and Gurunathan S. 2017. Effects of Silver Nanoparticles on Multiple Drug-Resistant Strains ofStaphylococcus aureusandPseudomonas aeruginosafrom Mastitis-Infected Goats: An Alternative Approach for Antimicrobial Therapy. International Journal of Molecular Sciences 18:569. DOI: 10.3390/ijms 18030569

Zhang L, Gu FX, Chan JM, Wang AZ, Langer RS, and Farokhzad OC. 2008. Nanoparticles in medicine: therapeutic applications and developments. Clinical Pharmacology \& Therapeutics 83:761-769. DOI: 10.1038/sj.clpt.6100400

Zhao G, and Jr SS. 1998. Multiple parameters for the comprehensive evaluation of the susceptibility of Escherichia coli to the silver ion. Biometals 11:27-32. DOI:10.1023/A:1009253223055 
Table $\mathbf{1}$ (on next page)

Oligonucleotide primers used in this study 


\begin{tabular}{cc}
\hline Primer name & Oligonucleotide (5'-3') \\
\hline rt-16s-f & TTTGAGTTCCCGGCC \\
rt-16s-r & CGGCCGCAAGGTTAA \\
rt-bcsA-f & GATGGTACAAATCTTCCGTC \\
rt-bcsA-r & ATCTTGGAGTTGGTCAGGCT \\
rt-csgA-f & AGCGCTCTGGCAGGTGTTGT \\
rt-csgA-r & GCCACGTTGGGTCAGATCGA \\
rt-fliC-f & CCTGAACAACACCACTACCA \\
rt-fliC-r & TGCTGGATAATCTGCGCTTT \\
rt-motA-f & GGCAATAATGGCAAAGCGAT \\
rt-motA-r & CAGCGAAAACATCCCCATCT \\
rt-wcaF-f & TCTCGGTGCCGAAAGGGTTC \\
rt-wcaF-r & ATTGACGTCATCGCCGACCC \\
rt-fimA-f & TGCTGTCGGTTTTAACATTC \\
rt-fimA-r & ACCAACGTTTGTTGCGCTAC \\
\hline
\end{tabular}

1 


\section{Figure 1}

Colony-forming unit assays of E. coli strain ECDCM1 incubated with Qe, Ag NPs, and QA NPs.

(A) Qe; (B) Ag NPs; (C) QA NPs. The survival rate of the control group without exposure to Qe, Ag NPs, or QA NPs agents was designated $100 \%$. The colony counts of the test group cultured with Qe, Ag NPs, or QA NPs at different concentrations were all compared to those of the control group with no exposure to Qe, Ag NPs, or QA NPs. Error bars indicate standard deviations. Double asterisks (**) represent means significantly different from the control group with no exposure to Qe, Ag NPs, or QA NPs $(P<0.01)$. 


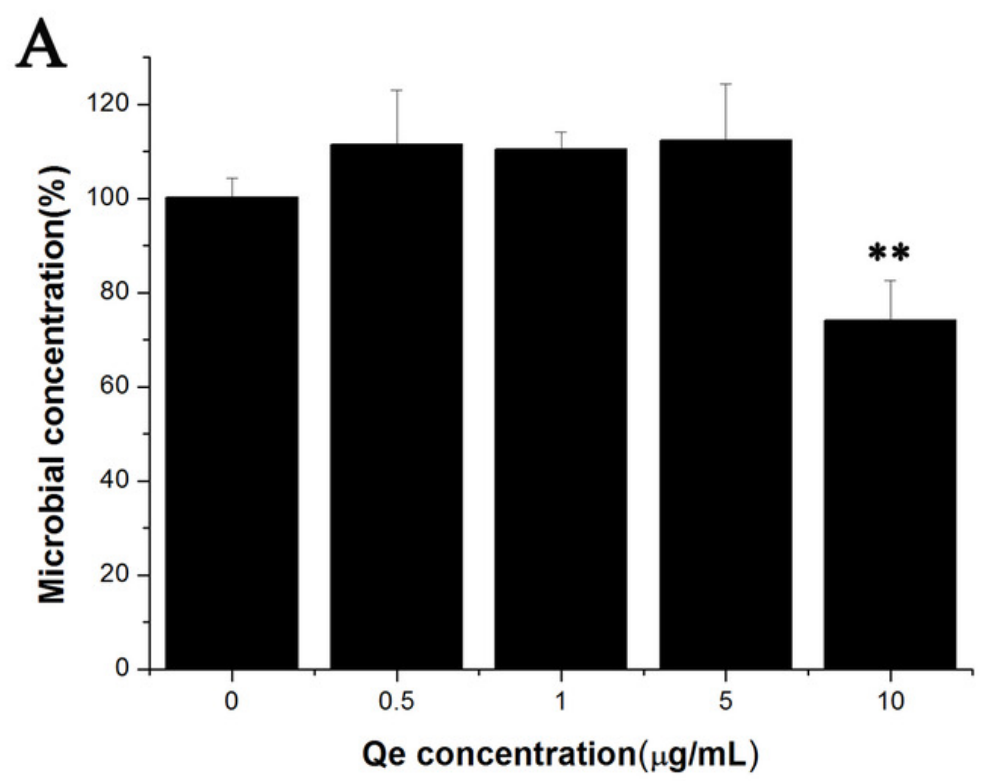

B
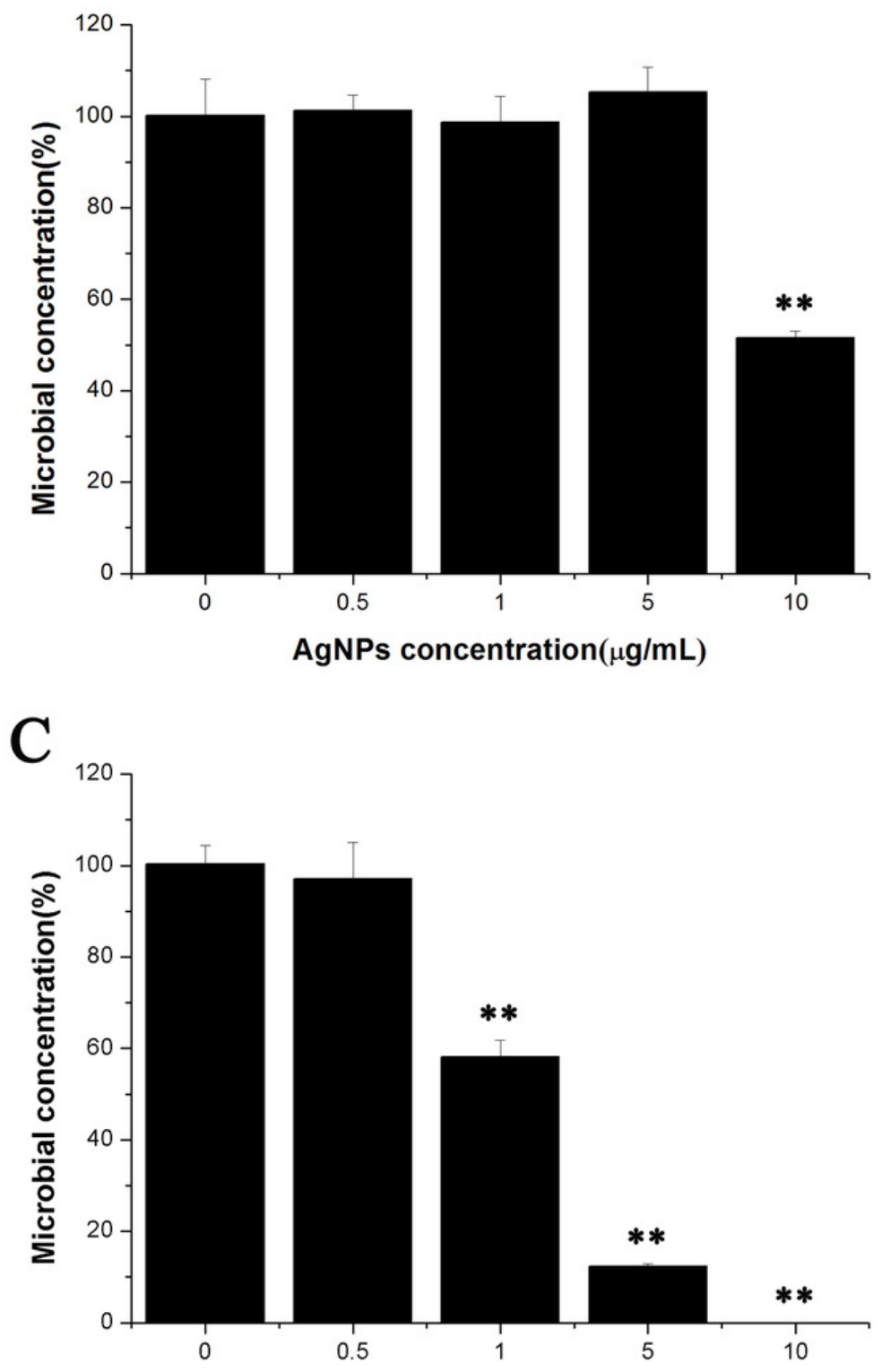

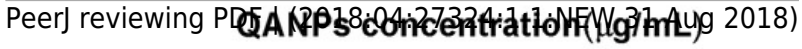




\section{Figure 2}

Effect of Qe, Ag NPs, and QA NPs on biofilm formation of $E$. coli strain ECDCM1.

(A) photographs of cells adhering to $15 \mathrm{~mm} \times 150 \mathrm{~mm}$ glass tubes: (a) Qe; (b) Ag NPs; (c) QA NPs; (B) a crystal violet assay and the biofilm quantity being dissolved in $33 \%$ glacial acetic acid was measured by optical density at 492 nm: (a) Qe; (b) Ag NPs; (c) QA NPs. Error bars indicate standard deviations. The results represent a mean of 3 independent experiments. Double asterisks $\left({ }^{* *}\right)$ represent means significantly different from the control group with no exposure to Qe, Ag NPs, or QA NPs ( $\mathrm{P}<0.01)$; an asterisk (*) represents means significantly different from the control group with no exposure to Qe, Ag NPs, or QA NPs $(P<0.05)$. 
A

a)

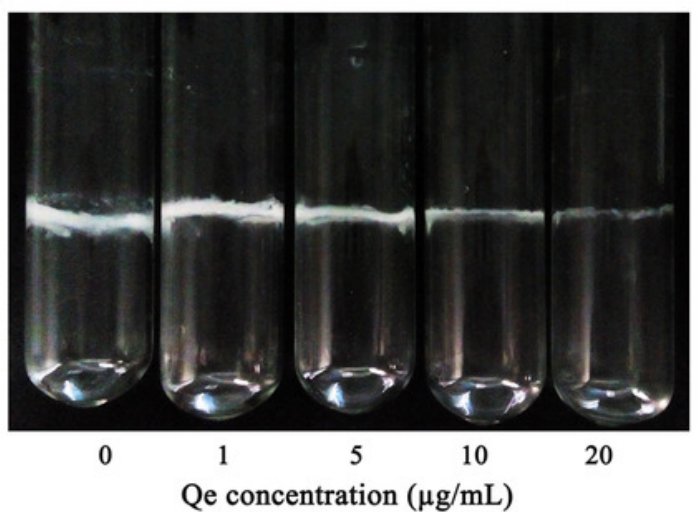

b)

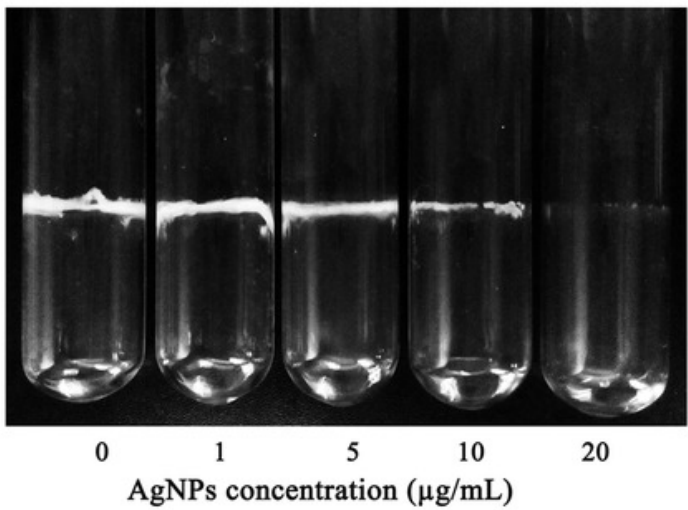

c)

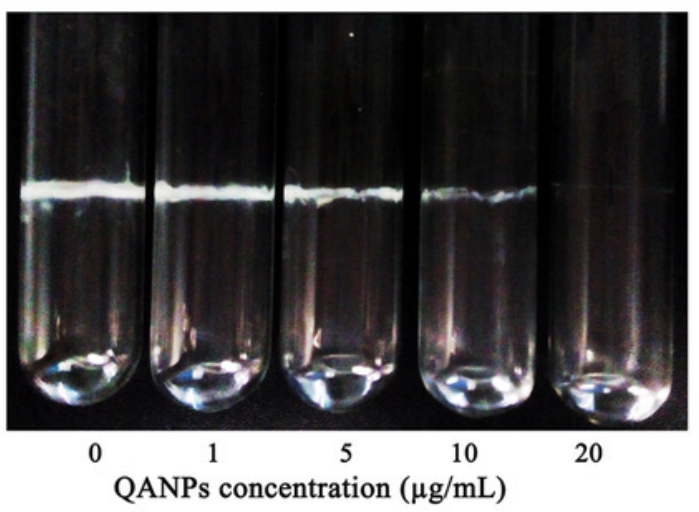

B
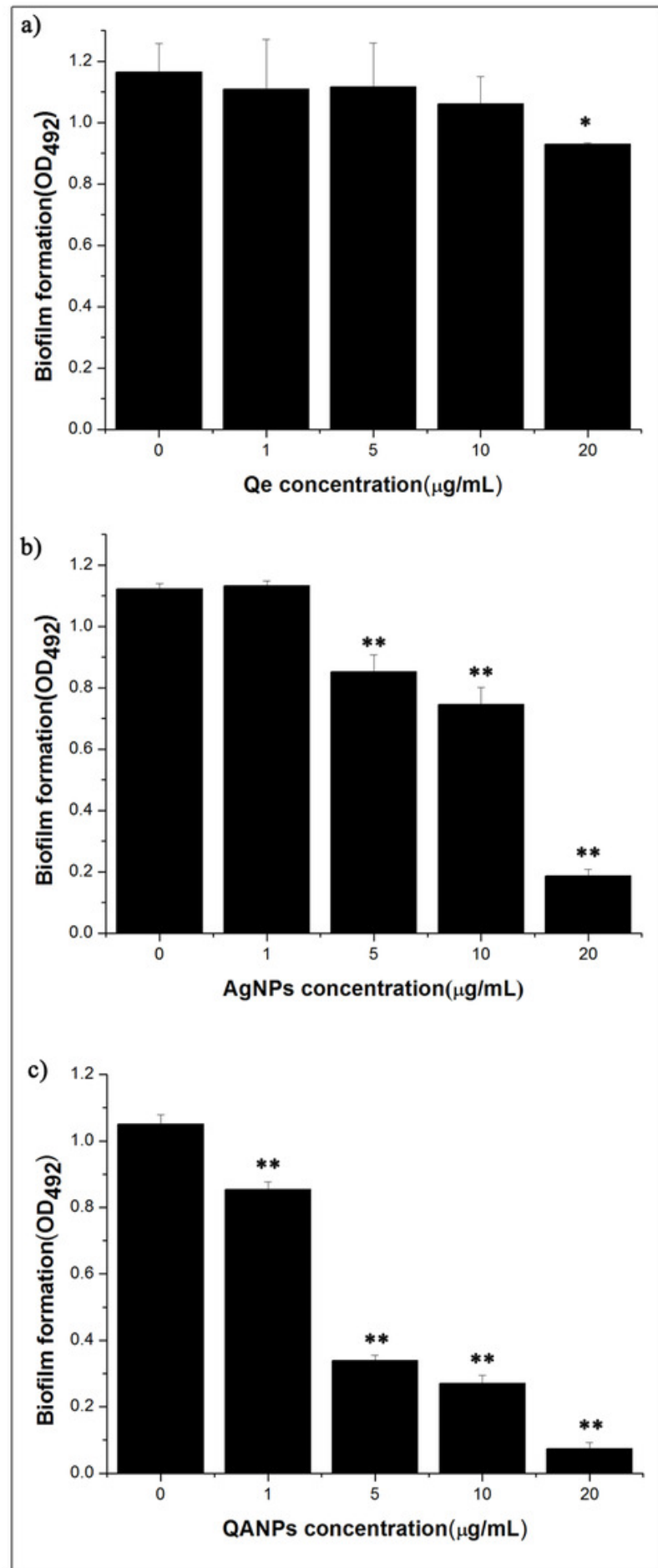


\section{Figure 3}

Effect of Qe, Ag NPs, and QA NPs on biofilm integrity of E. coli strain ECDCM1 were monitored by SEM.

(A) the control group with no antimicrobial agents; (B) E. coli strain ECDCM1 with exposure to

Qe; (C) E. coli strain ECDCM1 with exposure to Ag NPs; (D) E. coli strain ECDCM1 with exposure to QA NPs.

*Note: Auto Gamma Correction was used for the image. This only affects the reviewing manuscript. See original source image if needed for review.
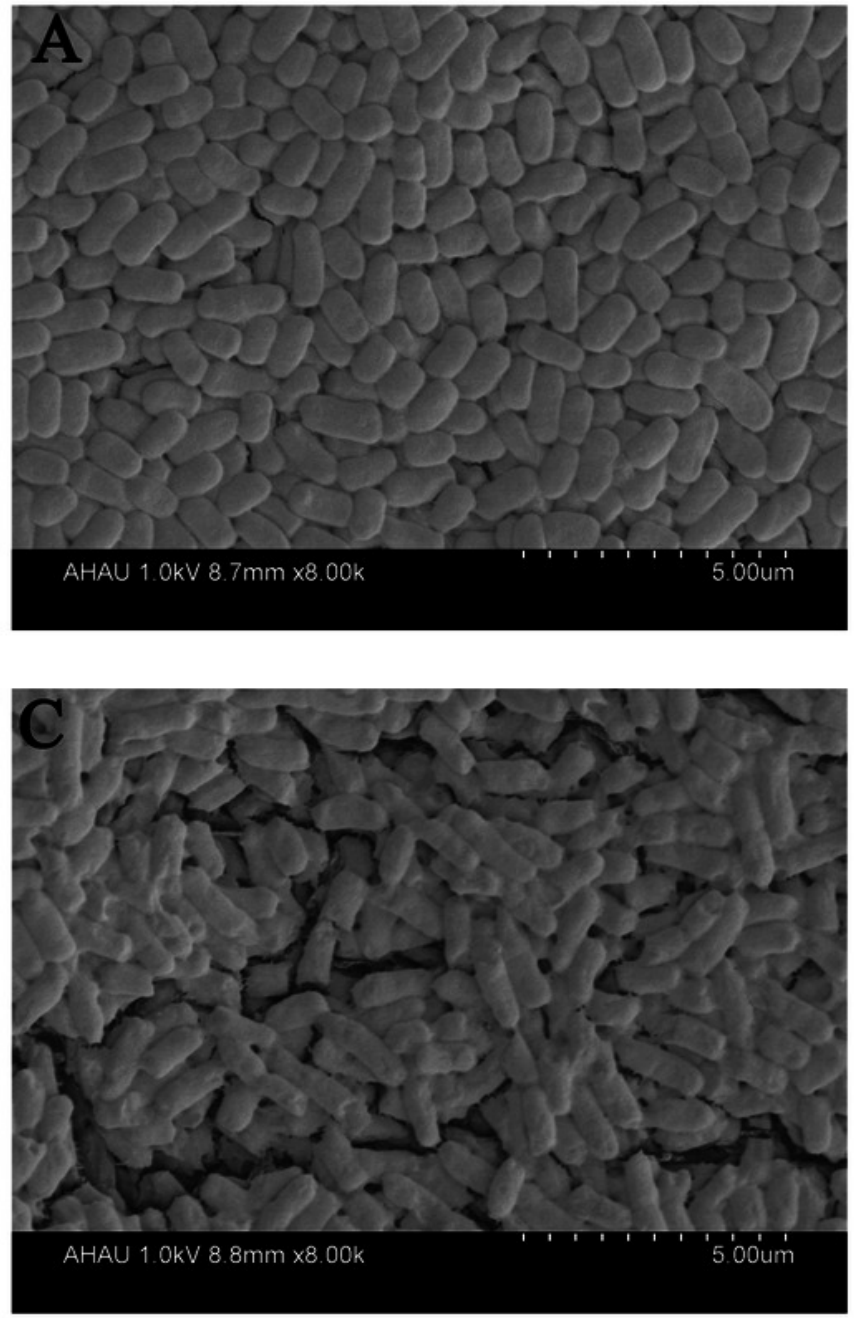
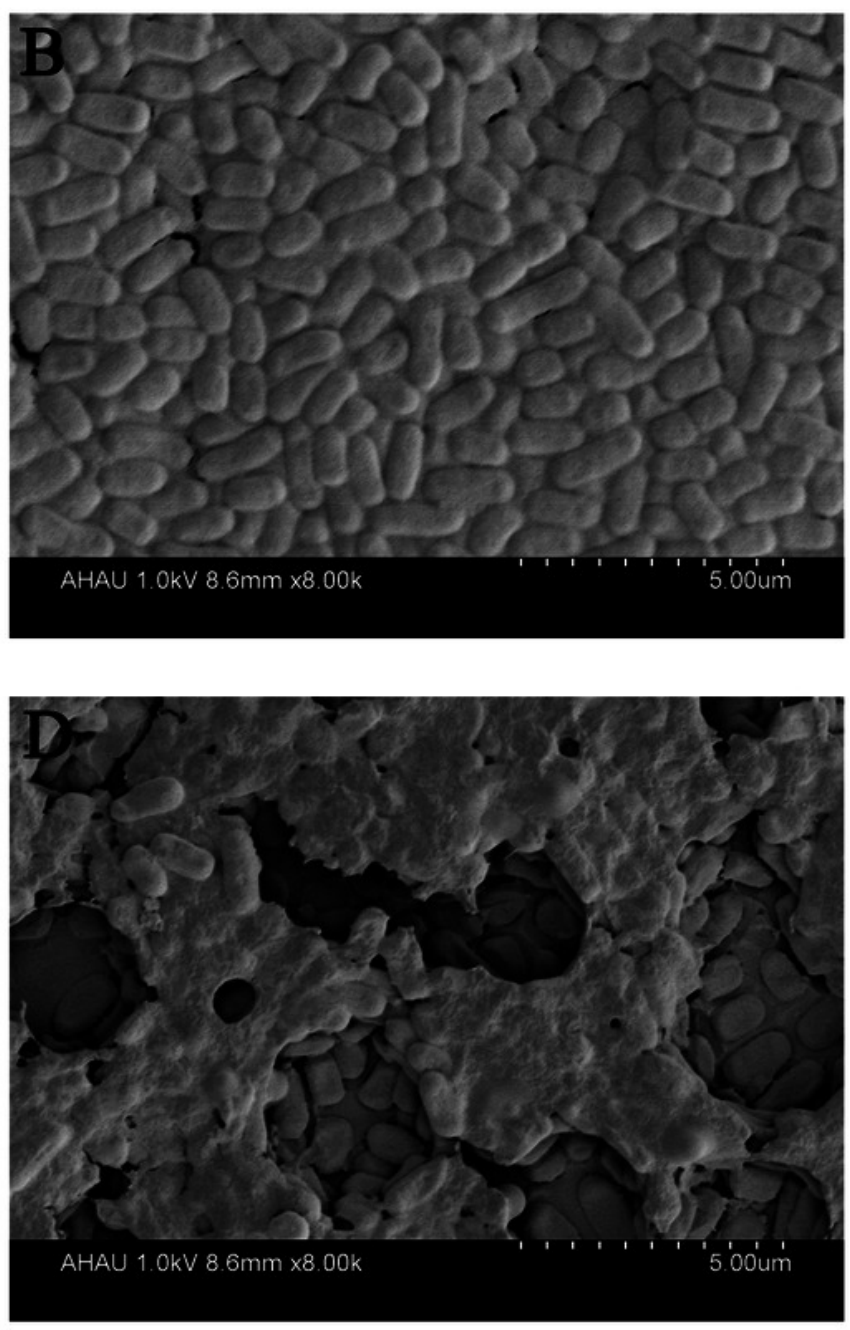


\section{Figure 4}

Comparative measurement of transcription (cDNA abundance) of the biofilm-associated genes in E. coli strain ECDCM1

Relative transcript levels of $b c s A, c s g A, f l i C, w c a F, \operatorname{mot} A$, and fimA were determined by realtime RT-PCR in E. coli strain ECDCM1 cultured without or with Qe, Ag NPs, or QA NPs. Error bars indicate standard deviations. Double asterisks $\left({ }^{* *}\right)$ represent means significantly different from control group with no antimicrobial agents $(P<0.01)$.

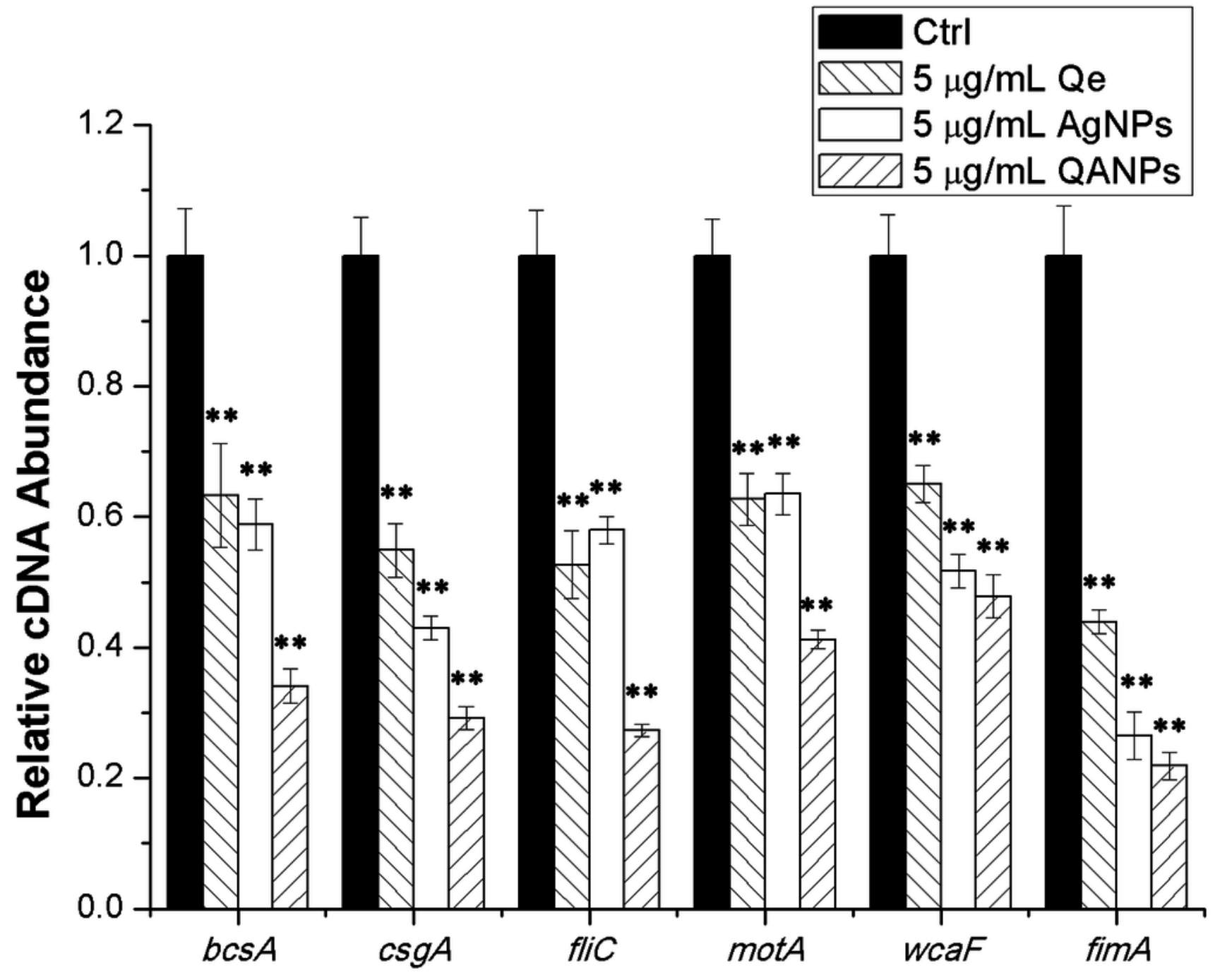

